\title{
A review of cognitive orientation to daily occupational performance with stroke
}

\author{
Si-Nae Ahn \\ Department of Occupational Therapy, Yeoju Institute of Technology, Yeoju, Republic of Korea
}

Objective: The self-decisions of the client regarding the meaningful work as a therapeutic approach of client-orientation. The Cognitive Orientation to daily Occupational Performance (CO-OP) is an occupation-oriented problem-solving approach. The purpose of this study was to describe the goals and intervention protocols of CO-OP in those affected by stroke.

Design: A systematic review.

Methods: Using EBSCOhost, PubMed, and ProQuest databases, we searched studies published in the past decade that utilized the CO-OP intervention. An initial search revealed 71,171 potential articles. After applying our search criteria to screen the titles, abstracts, and full-text, we included 7 articles that met our inclusion and exclusion criteria. In this study, we used the patient, intervention(s), comparison, outcome method to analyze the 7 selected studies. We analyzed the frequency of goals and intervention protocols.

Results: Seven articles met our selection criteria; these studies included participants with an almost normal cognitive function from inpatient and outpatient rehabilitation facilities. CO-OP was used for 237 goals; the most used goal was the instrumental activities of daily living. The training procedure used 3 types of self-selecting goals in the activities. One of the goals was not trained, but was only evaluated to determine the generation effect. The most common outcome measurements included the Canadian Occupational Performance Measure and the Performance Quality Rating Scale.

Conclusions: This research provided information about the effectiveness of CO-OP and selecting the correct evaluation tool to assess the efficiency of the intervention. This study suggests that treatment with CO-OP in occupational therapy is effective and that it outlines common protocols.

Key Words: Cognition, Occupations, Stroke

\section{Introduction}

Most occupational therapies applied to the stroke population in the past consisted of bottom-up approaches. However, recent occupational therapies utilize a client-centered top-down approach and an occupation-based intervention with the purpose of 'activity and participation' in the clinical area [1]. The top-down approach selects goals based on occupational performance; the therapist then educates or trains the patient directly to achieve the occupational goals by considering the environment of the client $[2,3]$. The top-down approach grants motivation because of the self-decisions of the client regarding the meaningful work as a therapeutic approach of client-orientation; thus, the client can actively participate in the treatment. Furthermore, there is the advantage of high satisfaction with treatment [4]. The Cognitive Orientation to daily Occupational Performance (CO-OP) is well known for clinical usability in occupational therapy.

The CO-OP approach is an occupation-oriented problem-solving approach based on the learning theory, motor learning theory, and cognitive theory $[5,6]$. CO-OP uses a meta-cognitive strategy, an approach that promotes self-direction and self-monitoring to identify one's problems by

Received: 7 September, 2017 Revised: 21 November, 2017 Accepted: 26 November, 2017

Corresponding author: Si-Nae Ahn

Department of Occupational Therapy, Yeoju Institute of Technology, 338 Sejong-ro, Yeoju 12652, Republic of Korea

Tel: 82-33-760-2496 Fax: 82-33-760-2496 E-mail: otlovesn@gmail.com

(c) This is an Open-Access article distributed under the terms of the Creative Commons Attribution Non-Commercial License (http://creativecommons.org/licenses/ by-nc/4.0) which permits unrestricted non-commercial use, distribution, and reproduction in any medium, provided the original work is properly cited.

Copyright $\odot 2017$ Korean Academy of Physical Therapy Rehabilitation Science 
combining a cognitive strategy to detailed occupational training. Therefore, the cognitive strategy takes care of the central role in the learning process of performance of occupation and motor skills $[7,8]$. The client learns global problem-solving strategies [5]. The global problem-solving strategy, a meta-cognitive strategy of CO-OP, is executed in the process of 'GOAL-PLAN-DO-CHECK'. This process allows the subject to set goals (GOAL) and allows the therapist to guide in finding a detailed plan to achieve that goal (PLAN). Next, the subject executes the plan (DO) and continuously checks whether the goal is accomplished. Finally, the subject verifies whether a modification is crucial to the goal (CHECK) [5].

The CO-OP approach was developed in early 2000 for children suffering from developmental coordination disorder (DCD). The effectiveness of this approach is well-documented, and this technique specifically increases occupational performance based on motor function in DCD children using a cognitive strategy [9]. Recently, CO-OP has been applied to adults of various diagnosis groups including traumatic brain damage and stroke. The CO-OP method is mainly used in stroke studies. In stroke studies, CO-OP is associated with changes in occupational performance, increases in motor, cognitive, psychosocial abilities, and motivation. Additionally, an overall positive effect has been reported. However, to date, no study has established the success of the CO-OP approach regarding the protocol execution method of the CO-OP.
Therefore, this research analyzed published studies that executed protocols utilizing the CO-OP approach in individuals with stroke. Additionally, we analyzed the self-choosing goals and outcome measurement tools used to compare the effectiveness of the experimental results. Finally, we analyzed the application of CO-OP and self-selecting goal activities (set by the subject) to provide useful information for applying this method for clinical purposes. These results were combined to identify the effectiveness of the development of the cognitive strategy and the occupational performance of individuals with stroke.

\section{Methods}

\section{Searching process}

In this study, we conducted a computerized search encompassing a 10-year span (from 2005 to 2015) via EBSCOhost, PubMed, and ProQuest. We used the search terms "Cognitive Orientation to daily Occupational Performance" and "CO-OP". We included human studies, using CO-OP for individuals with stroke that were published in English in the experimental design. An initial search revealed 71,171 potential articles. After applying our search criteria to screen the titles, abstracts, and full-texts, we included 7 articles that met our inclusion and exclusion criteria (Figure 1).

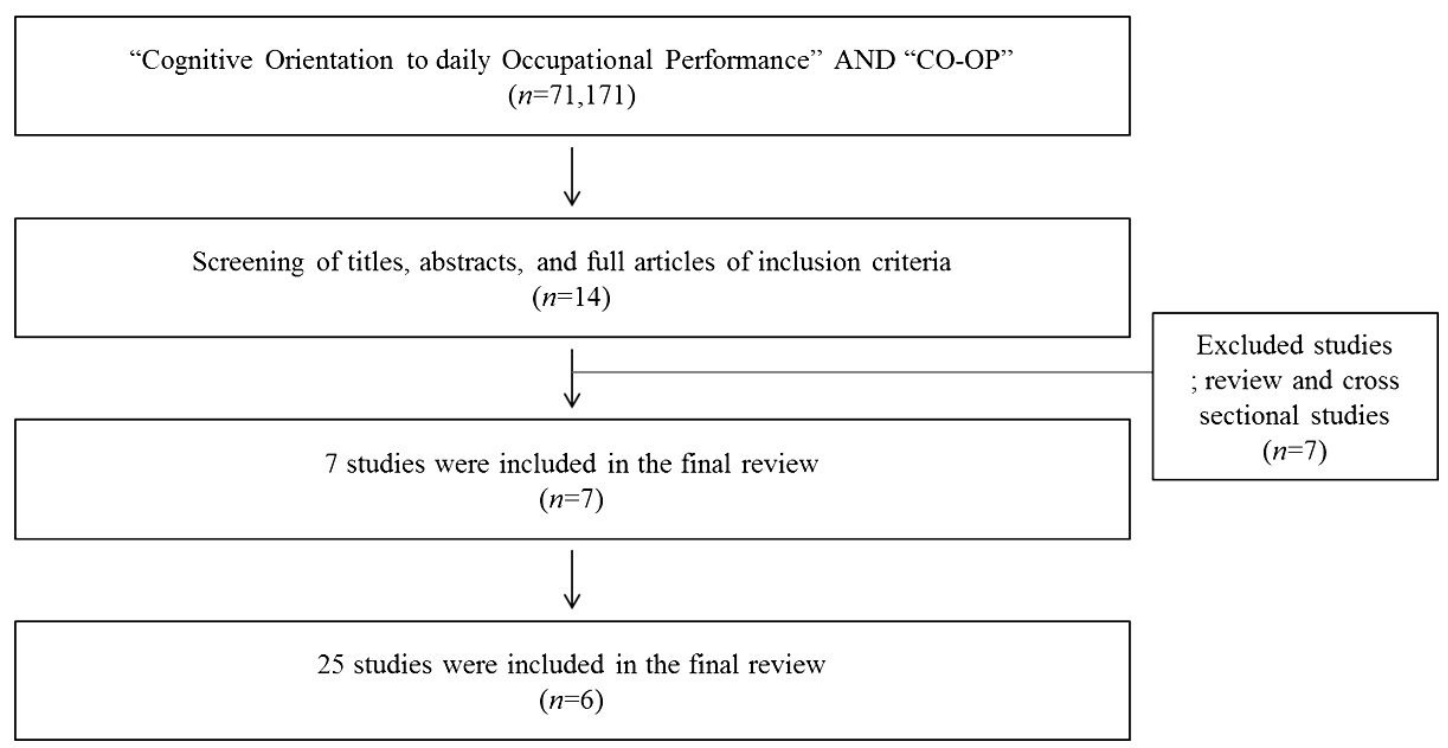

Figure 1. Flow diagram for literature search and selection process. CO-OP: Cognitive Orientation to daily Occupational Performance. 

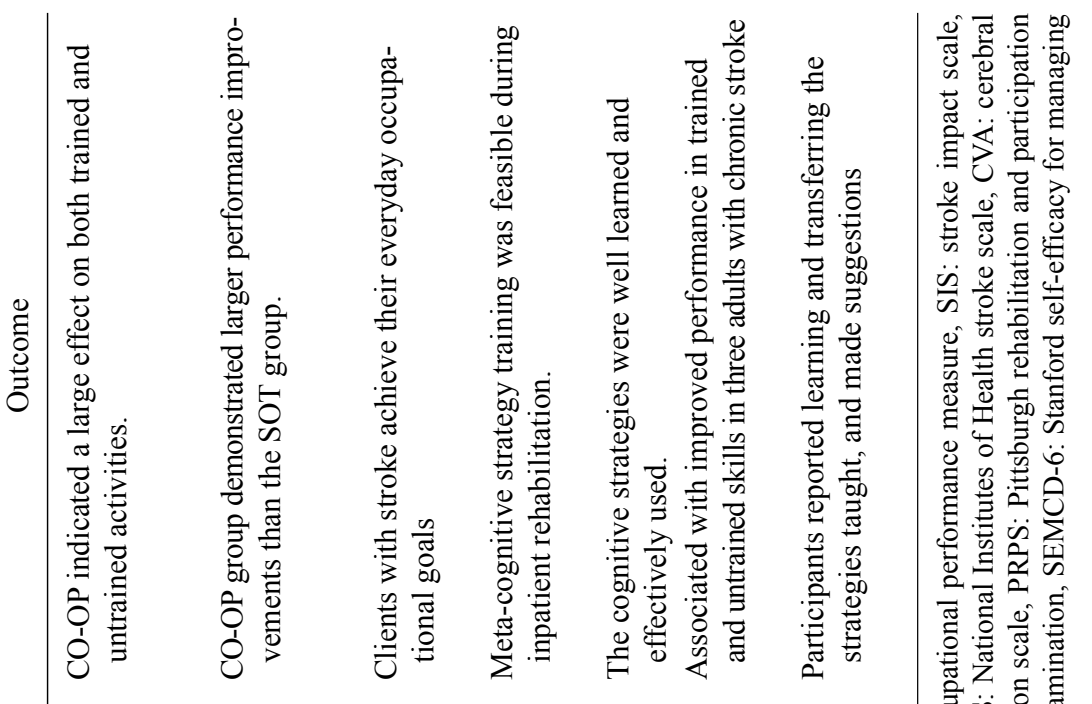

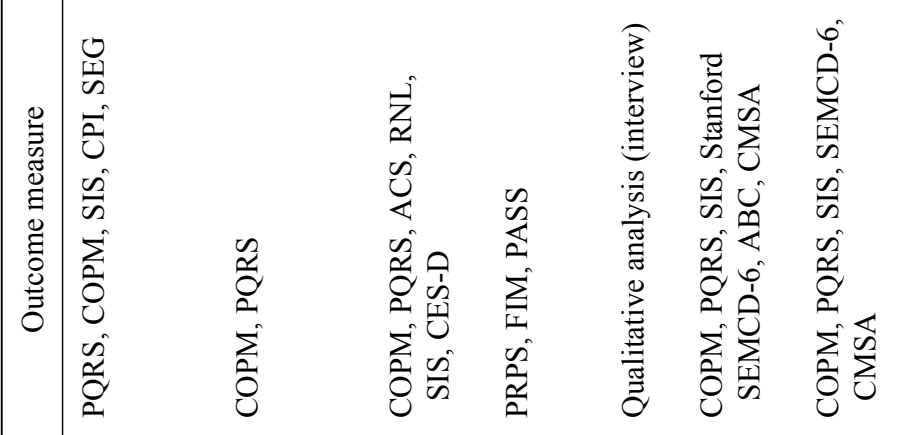

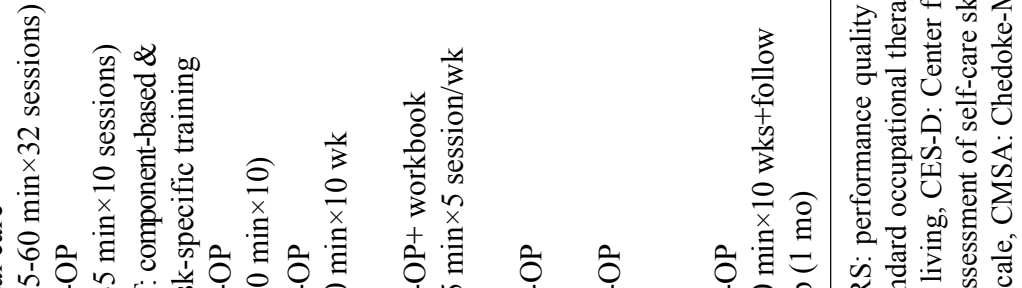

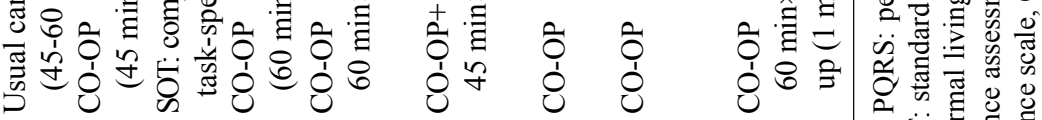
Iิ 于

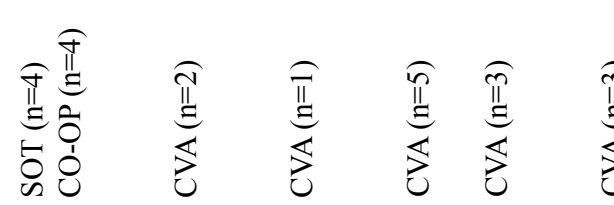

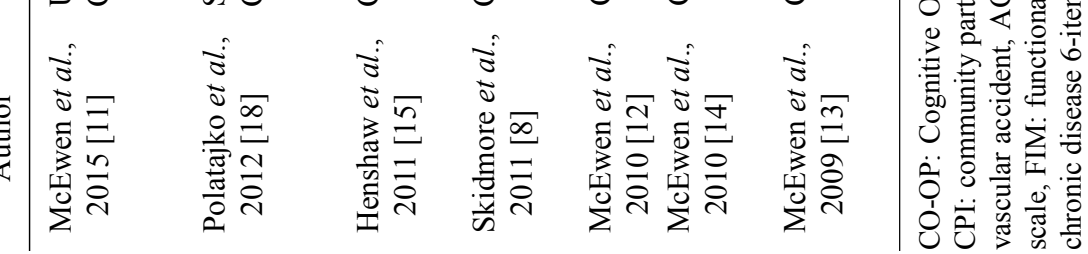




\section{Data collection}

Our inclusion criteria included studies that (1) enrolled participants with stroke and/or hemiparesis due to stroke, (2) used CO-OP, which is the CO-OP through intervention method, (3) were published in English, (4) were published between 2005 and 2015, and (5) provided the full-text article. Our exclusion criteria included research that did not provide an intervention (review articles and cross-sectional studies).

\section{Analysis methods}

In this study, we used the patient, intervention(s), comparison, outcome (PICO) method to analyze the 7 selected studies. "P" represents the population, including information about the characteristics of the subjects in each study. "I" represents the intervention and describes how the intervention was executed. "C" represents comparison and analyzes the measurement tool used to compare the intervention. "O" is the outcome, which analyzes the results from performing the intervention.

\section{Classification of intervention goals for $\mathrm{CO}-\mathrm{OP}$}

When executing the CO-OP approach, the first stage is to select a goal that the subject desires. Three goal activities desired by the subject and guardian are selected, and training on these goals is executed. Thus, this research examined the goal decided by the subject to achieve the CO-OP approach. We analyzed frequency according to performance skills and occupational domain corresponding to the occupational therapy practice framework (OTPF) domain and process [10].

This classification assessed the resulting measurement tool used in the research that executed the CO-OP approach and its frequency. Additionally, we compared the evaluation tools used when executing the CO-OP approach and other interventions.

\section{Results}

According to our selection criteria, 7 studies met the in clusion criteria and were analyzed using the PICO method (Table 1).

During the CO-OP approach, each participant sets a training goal. In the 7 selected studies, the number of goals set by the participants in the CO-OP approach was 237 [11-15]. In the selected seven studies, the selection criteria of the participants were applied in a similar manner. All participants were stroke survivors with normal cognitive function. In addition, participants who did not have aphasia or language disorders were included.

We divided these goals into repetitive items according to performance, skill, and occupation domain corresponding to the domain and process. Once these items were separated, we analyzed the frequencies (Table 2). Seventy-one goals were affiliated with activities of daily living (ADL) of the occupational domain including dressing, putting on earrings, using the hemiplegic hand to clip fingernails, etc. There were 101 goal activities affiliated with instrumental activities of daily living (IADL) including making coffee, handwriting, reading a book, bicycling, gardening, using a computer, taking medicine, etc. In the leisure category, there were 17 goals including attending a synagogue, traveling to see family, etc. There were 18 goals activities affiliated with working including handyman work, applying for a job, office activities, etc. Additionally, there were 30 goals affiliated with performance skills including transferring an object, walking, maintaining a standing position, etc.

To compare the effect of the CO-OP intervention in the 7 selected studies, we assessed the frequency of each outcome measurement tool (Table 3). The evaluation tools most fre-

Table 2. Goals for each of the domain of occupational therapy

$(\mathrm{N}=237)$

Domain of occupational therapy

Occupations

Activities of daily living $(\mathrm{n}=71)$

Instrumental activities of daily living $(\mathrm{n}=101)$

Leisure $(\mathrm{n}=17)$

Works $(\mathrm{n}=18)$

Performance skills $(n=30)$
Dressing, putting on earrings, putting on a bracelet, putting on jacket, use hemiplegic hand to clip finger nails, toileting et al.

Reading book, making coffee, praying, bicycling, swimming breaststroke, gardening, managing daily schedule, using camera, using computer, taking medicine et al. Synagogue, traveling to see family et al. Handyman work, apply for job, office activities et al. Transfer object, walking, maintaining standing position, remembering during cooking, concentration et al. 
Table 3. Frequency of assessment tool used CO-OP approach $(\mathrm{N}=12)$

\begin{tabular}{lc}
\hline \multicolumn{1}{c}{ Assessment tool } & $\mathrm{n}$ \\
\hline COPM, PQRS & 5 \\
SIS & 4 \\
SEMCD-6, CMSA & 2 \\
ACS, RNL, CES-D, FIM, PASS, ABC, PRPS, CPI, SEG & 1 \\
\hline
\end{tabular}

CO-OP: Cognitive Orientation to daily Occupational Performance, COPM: Canadian occupational performance measure, PQRS: performance quality rating scale, SIS: stroke impact scale, SEMCD-6: Stanford self-efficacy for managing chronic disease 6-item scale, CMSA: Chedoke-McMaster stroke assessment, ACS: activity card sort, RNL: reintegration to normal living, CES-D: Center for Epidemiological Studies depression scale, FIM: functional independence measurement, PASS: performance assessment of self-care skills, ABC: activity-specific balance confidence scale, PRPS: Pittsburgh rehabilitation and participation scale, CPI: community participation index, SEG: self-efficacy gauge.

quently used included the Canadian occupational performance measure (COPM) and performance quality rating scale (PQRS), which were each used 5 times in the CO-OP studies. The second most commonly used measure was the stroke impact scale applied in 4 studies. The Stanford self-efficacy for managing chronic disease 6-item scale and Chedoke-McMaster stroke assessment were applied 2 times, whereas the activity card sort, reintegration to normal living, Center for Epidemiological Studies depression scale, functional independence measurement, performance assessment of self-care skills, activity-specific balance confidence scale, and Pittsburgh rehabilitation and participation scale were each used once.

\section{Discussion}

The CO-OP strategy is used for problem solving by identifying a problem and setting a goal to assess one's performance in achieving the set goal. Previous studies have reported improved awareness in problem solving and implementing the meta-cognitive strategy, thereby reducing performance errors and improving performance skills in individuals who have suffered a stroke. As a result of analyzing 7 experimental studies, which all used the CO-OP approach for stroke participants, we noted that all subjects exhibited improvements in achieving their self-selected goals. Additionally, occupational performance and related functions increased. Specifically, the quality of life and satisfaction increased as a result of the CO-OP intervention.

A point to focus on is that there were only 2 group studies that applied the CO-OP intervention, and all of the other studies were case studies or case series. Since the CO-OP is typically provided individually, after setting a goal accordingly to the request and environment of an individual, group research using the CO-OP is difficult.

When we classified the client-goals according to the OTPF domain and process for the training of CO-OP approach, goal activities corresponding to IADL from the occupation domain were the most frequent. As most of the participants in the CO-OP approach had normal cognitive function, performance of IADLs were greater than ADLs, as most of the time was spent on eating, managing family, and maintaining health. There were a few performance skill goals, most regarding body function. Since most persons with stroke have limited body function, due to hemiparalysis, several goals are centered on the transfer of objects or walking.

In previous studies, the $\mathrm{CO}-\mathrm{OP}$ approach has been generalized and transferred consistently [9,11]. For this reason, the cognitive and metacognitive elements are superimposed on task-based training. Through this process, participants learn to analyze their performance and develop strategies to overcome issues. Therapists guide their clients to develop performance strategies and to use these strategies to their advantage. In our study, the most frequently reported goals included IADL of occupations.

The most common evaluation tools used to assess the effectiveness of the CO-OP approach were the COPM and the $\mathrm{PQRS}$. The CO-OP approach allows the subject to select the desired goal with the training tailored to achieve this goal. The COPM evaluates the occupational performance ability of the client. This tool identifies issues in the occupational performance domain during the half-structural interview process with the therapist. The priority of the assessment is then classified by importance level, performance level, and satisfaction level, based on a 10-point scale $(1=$ cannot perform/satisfy, 10=perform/satisfy) [16]. For these reasons, the COPM is mostly commonly used to evaluate performance level and satisfaction level felt by the participants through self-report interviews. The PQRS also observes and measures the quality of performance through the structural definition of the therapist by allowing the client to decide on the goal activity [17]. The PQRS is very simple as it measures individual activity from a score of 1 (cannot perform) to 10 (perform very well) [18]. Thus, it is an appropriate measure to evaluate the qualitative change of performance, which is why this method is frequently used. 
In this study, there were clinical opinions on the need to provide necessary information to the application of CO-OP approach to stroke in a clinical setting. Furthermore, when taken together, the findings of advanced studies that executed the CO-OP approach on the stroke population indicated increased physical function and daily activity performance through problem-solving strategies to develop cognitive strategies and also the trained goal activities. However, only 7 studies met our inclusion criteria, making it difficult to generalize our results. More randomized controlled trials are needed to determine the effectiveness of the CO-OP approach.

This research provided information about the effectiveness of $\mathrm{CO}-\mathrm{OP}$ and selecting the correct evaluation tool to assess the efficiency of the intervention. Additionally, the CO-OP approach improved the occupational performance ability for daily living. By providing individuals opportunities to solve problems on their own, CO-OP can help increase functional individuality in real life. This study provides considerable insight into the CO-OP application. The present study recommends the CO-OP approach and outlines standard evaluation tools.

\section{Conflict of Interest}

The author declared no potential conflicts of interest with respect to the authorship and/or publication of this article.

\section{References}

1. Mandich AD, Polatajko HJ, Macnab JJ, Miller LT. Treatment of children with Developmental Coordination Disorder: what is the evidence? Phys Occup Ther Pediatr 2001;20:51-68.

2. Doucet BM, Woodson A, Watford M. Moving toward 2017: progress in rehabilitation intervention effectiveness research. Am J Occup Ther 2014;68:e124-48.

3. Sansonetti D, Hoffmann T. Cognitive assessment across the continuum of care: the importance of occupational performance-based assessment for individuals post-stroke and traumatic brain injury. Aust Occup Ther J 2013;60:334-42.

4. O'Brien JC, Bergeron A, Duprey H, Olver C, Onge H. Children with disabilities and their parents' views of occupational participation needs. Occup Ther Ment Health 2009;25:164-80.

5. Polatajko HJ, Mandich AD, Miller LT, Macnab JJ. Cognitive ori- entation to daily occupational performance (CO-OP): part II--the evidence. Phys Occup Ther Pediatr 2001;20:83-106.

6. Polatajko HJ, Mandich A. Enabling occupation in children: the cognitive orientation to daily occupational performance (CO-OP) approach. Ottawa, ON: CAOT Publications ACE; 2004.

7. Missiuna C, DeMatteo C, Hanna S, Mandich A, Law M, Mahoney W, et al. Exploring the use of cognitive intervention for children with acquired brain injury. Phys Occup Ther Pediatr 2010;30:205-19.

8. Skidmore ER, Holm MB, Whyte EM, Dew MA, Dawson D, Becker JT. The feasibility of meta-cognitive strategy training in acute inpatient stroke rehabilitation: case report. Neuropsychol Rehabil 2011;21:208-23.

9. Skidmore ER, Whyte EM, Butters MA, Terhorst L, Reynolds CF 3rd. Strategy training during inpatient rehabilitation may prevent apathy symptoms after acute stroke. PM R 2015;7:562-70.

10. American Occupational Therapy Association. Occupational therapy practice framework: domain and process ( $3 \mathrm{rd} \mathrm{ed}$ ). Am J Occup Ther 2014;68(Suppl 1):S1-48.

11. McEwen S, Polatajko H, Baum C, Rios J, Cirone D, Doherty M, et al. Combined cognitive-strategy and task-specific training improve transfer to untrained activities in subacute stroke: an exploratory randomized controlled trial. Neurorehabil Neural Repair 2015;29:526-36.

12. McEwen SE, Polatajko HJ, Davis JA, Huijbregts M, Ryan JD. 'There's a real plan here, and I am responsible for that plan': participant experiences with a novel cognitive-based treatment approach for adults living with chronic stroke. Disabil Rehabil 2010;32:540-50.

13. McEwen SE, Polatajko HJ, Huijbregts MP, Ryan JD. Exploring a cognitive-based treatment approach to improve motor-based skill performance in chronic stroke: results of three single case experiments. Brain Inj 2009;23:1041-53.

14. McEwen SE, Polatajko HJ, Huijbregts MP, Ryan JD. Inter-task transfer of meaningful, functional skills following a cognitive-based treatment: results of three multiple baseline design experiments in adults with chronic stroke. Neuropsychol Rehabil 2010;20:541-61.

15. Henshaw E, Polatajko H, McEwen S, Ryan JD, Baum CM. Cognitive approach to improving participation after stroke: two case studies. Am J Occup Ther 2011;65:55-63.

16. Carpenter L, Baker GA, Tyldesley B. The use of the Canadian occupational performance measure as an outcome of a pain management program. Can J Occup Ther 2001;68:16-22.

17. Martini R, Rios J, Polatajko H, Wolf T, McEwen S. The performance quality rating scale (PQRS): reliability, convergent validity, and internal responsiveness for two scoring systems. Disabil Rehabil 2015;37:231-8.

18. Polatajko HJ, McEwen SE, Ryan JD, Baum CM. Pilot randomized controlled trial investigating cognitive strategy use to improve goal performance after stroke. Am J Occup Ther 2012;66:104-9. 